\title{
Treatment of Benign Paroxysmal Positional Vertigo: An Approach Considering Patients' Convenience
}

\author{
Dae Bo Shim (i) \\ Department of Otorhinolaryngology, Myongji Hospital, Hanyang University College of Medicine, Goyang, Korea
}

Benign paroxysmal positional vertigo (BPPV) is the most common cause of peripheral vestibulopathy, with a lifetime prevalence of $2.4 \%$ [1]. The basic pathophysiology of BPPV is that otoconia dislodged from the macula of the utricle enter the semicircular canals and then cause vertigo and nystagmus when the head's position changes. The posterior canal is the most common area of BPPV, accounting for $60 \%$ to $90 \%$ of total BPPV cases, followed by the lateral canal and the anterior canal [2]. According to the clinical practice guideline for BPPV presented by the Association of American Otolaryngology-Head and Neck Surgery, the Dix-Hallpike test (DHT) and the modified Epley maneuver are strongly recommended for the diagnosis and treatment of posterior canal BPPV, respectively [3]. Recently, the rate of treatment success within 24 hours after a single treatment with the modified Epley maneuver was reported to be as high as $83.6 \%$ [4]. Considering both the success rate of the treatment and patients' convenience, the optimal timing for reassessment is 24 hours after treatment [4].

The DHT and the modified Epley maneuver, as examination and treatment methods for BPPV, should be performed in an accurate manner to ensure that the otoconia in the posterior canal can be moved maximally within the membranous labyrinth in the semicircular canal. For this reason, the DHT and the modified Epley maneuver can cause patients to experience side effects such as a falling sensation, anxiety, and discomfort (nausea or imbalance). Patients with BPPV naturally experience severe discomfort during diagnosis and treatment since motion change causes severe vertigo. Recently, some authors reported that a modified version of the DHT with a pillow under the shoulders to resolve patients' discomfort during the standard DHT had the same diagnostic effectiveness as the standard DHT [5]. Following the previous report, the authors reported that the therapeutic efficacy of the modified Epley maneuver with a pillow under the shoulders was also comparable to that of the standard modified Epley maneuver [6]. The authors also stated that this modi- fied method can be helpful for patients who have anxiety about the head-hanging position during treatment and can reduce both the force needed and the physical burden on clinicians because they no longer need to hold onto the patient's head. Therefore, that study is very meaningful in that it focuses on the convenience of both patients and clinicians during BPPV treatment.

However, the study of Lee et al. [6] had some limitations: (1) no evaluation of patients' subjective discomfort, (2) a relatively small population, and (3) a short-term follow-up period. In addition, if the size of the pillow is not chosen depending on the size of the patient's body, the patient may not be positioned properly for treatment.

To improve the accuracy and effectiveness of BPPV treatment, as well as the convenience of patients, therapeutic methods using devices such as virtual reality or inertial measurement units have also been recently been reported [7]. In the future, diagnostic and therapeutic procedures using these devices are expected to benefit both clinicians and patients with BPPV.

\section{CONFLICT OF INTEREST}

No potential conflict of interest relevant to this article was reported.

\section{ORCID}

Dae Bo Shim https://orcid.org/0000-0002-2331-5000

\section{REFERENCES}

1. von Brevern M, Radtke A, Lezius F, Feldmann M, ZieseT, LempertT, et al. Epidemiology of benign paroxysmal positional vertigo: a pop-

Copyright (C) 2020 by Korean Society of Otorhinolaryngology-Head and Neck Surgery.

This is an open-access article distributed under the terms of the Creative Commons Attribution Non-Commercial License (https://creativecommons.org/licenses/by-nc/4.0)

which permits unrestricted non-commercial use, distribution, and reproduction in any medium, provided the original work is properly cited. 
ulation based study. J Neurol Neurosurg Psychiatry. 2007 Jul;78(7): 710-5.

2. Kim JS, Zee DS. Clinical practice: benign paroxysmal positional vertigo. N Engl J Med. 2014 Mar;370(12):1138-47.

3. Bhattacharyya N, Gubbels SP, Schwartz SR, Edlow JA, El-Kashlan H, Fife T, et al. Clinical practice guideline: benign paroxysmal positional vertigo (update). Otolaryngol Head Neck Surg. 2017 Mar;156(3_ suppl):S1-47.

4. Song MH, Kong TH, Shim DB. Optimal reassessment time for treatment response in posterior canal benign paroxysmal positional vertigo. Laryngoscope. 2020 Feb;130(2):496-9.

5. Jeon EJ, Lee DH, Park JM, Oh JH, Seo JH. The efficacy of a modified Dix-Hallpike test with a pillow under shoulders. J Vestib Res. 2019;29(4):197-203.
6. Lee HJ, Jeon EJ, Lee DH, Seo JH.Therapeutic Efficacy of the modified Epley maneuver with a pillow under the shoulders. Clin Exp Otorhinolaryngol. 2020 Nov;13(4):376-80.

7. Tabanfar R, Chan HL, Lin V, Le T, Irish JC. Development and face validation of a Virtual Reality Epley Maneuver System (VREMS) for home Epley treatment of benign paroxysmal positional vertigo: a randomized, controlled trial. Am J Otolaryngol. 2018 Mar-Apr;39(2): 184-91.

Received October 7, 2020 Accepted October 21, 2020 